\title{
Mind Controlled Electric Power Steering
}

\author{
Adam DePalma 1 , Yue Guo ${ }^{2}$, Matt Leser ${ }^{3}$, Kevin Smith ${ }^{4}$, Dean Aslam ${ }^{5 *}$ \\ Electrical and Computer Engineering Department, Michigan State University, MI 48824, USA ${ }^{1-5}$
}

\begin{abstract}
In the United States, there are roughly 291,000 people living with a spinal cord injury as of 2019 [1]. Of these people, over $66 \%$ of them suffer from either incomplete or complete tetraplegia [1]. Tetraplegia, or more commonly known quadriplegia, is a term used to describe the inability to move or feel both the upper and lower parts of one's body, such as the arms, chests, legs, and feet [2] [3]. Adjusting to a new life for people who have lost the ability of both their arms and legs can be very difficult [4]. One of the activities lost from tetraplegia is the ability to drive a vehicle. In this work, we have considered brainwave activity alongside pose tracking software to control an electric motor and to simulate steering a vehicle. Controlling an electric motor represents the electric power steering system. Electric power steering systems work by determining how much torque needs to be input into a steering wheel to alter the direction a vehicle [5]. By combining two distinctive trends of total brainwave activity and the direction that a subject's head is facing, we study a potential solution to ease the lives of disabled Americans.
\end{abstract}

Keywords: Electroencephalogram, brainwave, driver monitoring, electric power steering, mind, tetraplegia, pose tracking.

\section{INTRODUCTION}

To be able to use one's own mind to control objects may seem like a superpower or fantasy to many, however, it is the subject of this research. The question asked in this paper is if someone can steer a vehicle using their mind. Challenging this question builds the potential of a new pathway allowing both tetraplegic people and people who may not have fine motor skills, the opportunity to drive once more. This question is challenged by analyzing brainwave activity and the role that the mind plays in it, and then coordinating the mind's role in brainwave activity to controlling a motor. The mind, defined earlier as what is responsible for one's thoughts and feelings [6], has recently been defined as an algorithm definable by electroencephalogram (EEG) data [7]. By using an EEG sensor, it is possible to measure brainwave activity. With the data collected from brainwave activity, patterns or distinct events may be analyzed and tied to controlling a motor through logical processes. Controlling a motor to spin in the direction that a driver is steering is the basis of how electric power steering systems work today. In modern designs, an electric control unit will process signals and logic to spin the motor in the appropriate direction to help a driver steer a vehicle [5]. Electric power steering systems are also utilized by receiving commands from other electric control units in a vehicle to perform automatic steering features, such as Toyota's "Intelligent Parking Assist System" [8]. Features like Toyota's rely on cameras to handle the processing of the surrounding environment [8]. These features offer luxury to drivers, but only in specific situations. If the cameras used to park the vehicle were replaced with the human mind, there is potential for a feature to be used in more environments. Instead of analyzing specific situations and executing the same maneuvers, the human mind may be able to analyze many situations and respond as people already do today. Through coordination of the brainwave activity of a human subject and motor control, the concept of steering a vehicle using one's mind is challenged.

\section{NEW AND EXCITING ASPECTS}

It may be noted that the idea of using an EEG to control a motor is not a brand-new concept. Automotive companies such as Nissan have already started research on using EEG headsets to help control the steering wheel in a vehicle [9]. Nissan has developed algorithms that will learn the patterns of the person driving the vehicle through an EEG headset [9]. The headset will read the signals from a person's brain and depending on the signals will attempt to guess how the driver is planning on moving the car [9]. For example, if the driver is planning on turning to the right, the headset will read this and will slowly start turning the steering wheel to the right to assist the driver [9].

Another automotive company that has moved its way in to the EEG vehicle scene is Jaguar [10]. Over the past few years, they have been working on a feature known as 'Mind Sense' [10]. The vehicle will monitor the brain waves of the pilot to help understand the condition of the driver [10]. An important parameter with Jaguar's research is that the EEG sensors are embedded through the steering wheel rather than as an EEG headset [10]. The different placements of the EEG sensor on the human body may have different results, and the algorithms that Jaguar has developed may be unique in their own regards. If the driver is appearing to fall asleep or is distracted by something, the vehicle will send warnings to get the drivers attention back onto the road [10]. 
Servo motor control is another area that is benefitting from EEG research. Being able to control servo motors with brain wave analysis can potentially open the door for many biomedical and generic motor control breakthroughs [11]. A team of researchers from the University of Baghdad were able to accurately control a servo motor to turn to a 90-degree position when specific EEG signal criteria was met, and then to turn back when the criteria was no longer met [11]. If this research can be built upon and multiple positions can be reached accurately, it may benefit consumers [11].

Other breakthroughs have been made in areas to allow tetraplegics to drive a vehicle without utilizing EEG sensor technology [12]. Racecar driver Sam Schmidt had suffered a career ending injury in 2000 that had left him unable to move his body from the neck down [12]. Schmidt was able to control a vehicle in 2017 using a tube that he may blow into or suck to accelerate the vehicle or use the brakes, voice commands to utilize turn signals, and head movement to steer the vehicle [12]. The vehicle allows Schmidt to regain much of the mobility he previously enjoyed, but the technology involved comes with a seven figure price tag, putting it out of reach of many Americans [12].

\section{METHODOLOGY}

The work is focused on creating a feature that would allow a driver to steer a vehicle using their mind. In the project, both a hardware and a simulation solution were tested. The hardware solution would be to accurately control the direction a motor is spun and when to spin it, this would represent the steering assist that is provided by electric power steering systems. The simulation solution would prove out the concept in a real time environment to show the feature working in a conceptual vehicle setting.

With the goal of establishing two proofs of concept, the authors began to study brainwave analysis. A NeuroSky Mindwave Mobile headset was selected for the EEG sensor used to collect data, as our mentor, Dr. Dean Aslam, had familiarity with the hardware. A subject would think about steering left or steering right and vary concentration levels. The thought processes continued as thinking steering right or thinking steering left for five seconds at a time. Then the subject would not think at all. This pattern was repeated for three minutes at a time for multiple samples. The supporting software for the NeuroSky headset would log the data payload to a comma separated value (CSV) file that could be read as the headset was logging. Brainwave signals in consideration were delta, theta, alpha, beta, and gamma signals. A summation of values across all brainwaves, named Total Brain Power, was considered as well. A graphical representation of the Total Brain Power collected by the data can be found in Fig. 1.

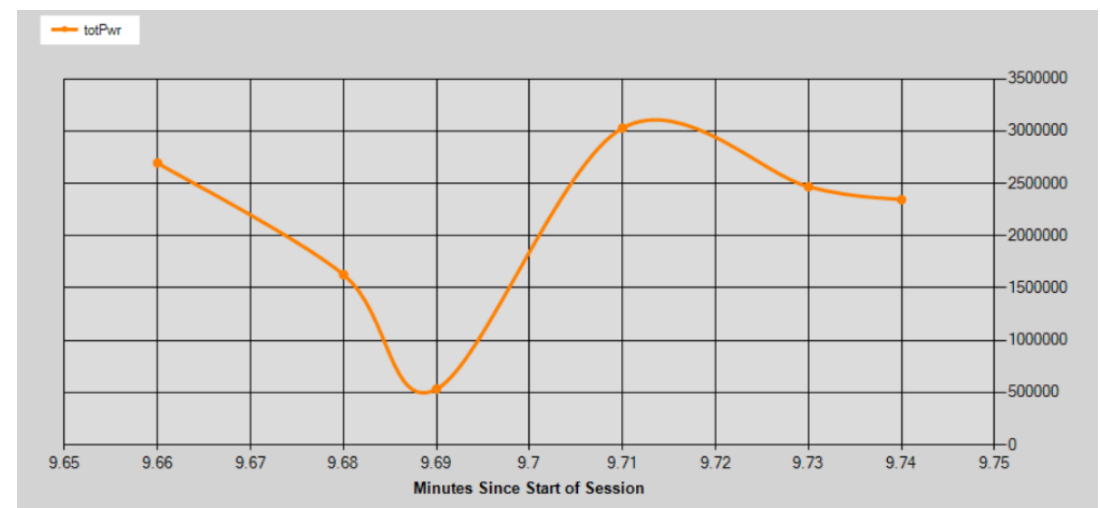

Fig. 1 Total Brian Power (measured in ASIC_EEG_POWER_INT [13]) over Time

First glances of the data showed that it was inconsistent and the different measured brain waves had a wide range of responses based on what the subject was doing. There was great difficulty in finding distinctive, repeatable, and accurate patterns within the data for any of the individually captured signals. The most noticeable trend that the authors were able to find is that in either deep thought, or muscle movement such as a blink or flexing of the forehead muscles, that the Total Brain Power would spike in EEG readings. In the graphical representation of Fig. 1, it is noticeable to see the spike in Total Brain Power on a blinking event. We decided to correlate the intent to steer when Total Brain Power exceeded a threshold. The proprietary algorithms used by NeuroSky translate the individual units of each brainwave signal into a unit called ASIC_EEG_POWER_INT [13]. This unit is similar to Volts-squared per Hertz but is transformed and scaled such that it does not have a simple linear correlation [13]. A Total Brain Power threshold of 3,000,000 was chosen to indicate that there was either intentional blinking, muscle movement, or deep thought. This value was chosen to attempt to avoid false positives as the authors progressed with testing. A second item that was troublesome with the data was the frequency of the payload from the headsets. The transmission rate of the NeuroSky Mindwave Mobile was roughly once per second. This slow messaging rate would prevent quick response from the system and allowed for the Total Brain Power threshold 


\title{
International Journal of Advanced Research in Computer and Communication Engineering
}

\author{
Vol. 9, Issue 8, August 2020
}

\section{DOI 10.17148/IJARCCE.2020.9802}

to be exceeded for roughly one second at a time before considering a new reading. There was now a need to add a directional parameter that could be coupled with the Total Brain Power exceeding a threshold.

It was decided that because the Total Brain Power threshold was not enough, a gaze tracking feature would be added. Gaze tracking can be used when a task is requested to gather more details about the intention of the request without prompting the user to provide those details [14]. This can be used as a reaction to a given command or used to predict a subsequent command [15]. The use case for this project was to control a motor to prove the same methodology of controlling a power steering unit. Even today, most gaze tracking is done by contact or non-contact systems. Contact systems attached to the user by a headset or, in the case of non-contact systems, can be a remote device [15]. For the project, using a laptop webcam provided a non-contact means to estimate gaze. To ensure accurate and repeatable results, the environment would need to be controlled since the camera needed to be calibrated with the user of the system and required limited head motion while testing [15]. Another option to achieve accurate results required multiple cameras and point light sources to avoid the calibration step [16]. However, an alternative that gave better accuracy, even with a single non-calibrated webcam, was using head pose estimation.

Head pose estimation is a system that approximates the orientation of a person's head with respect to the view of a camera. The camera does not need to be strictly calibrated as it can be estimated with some assumptions to reduce any bias from distortion [17]. More importantly, head pose estimation also provides a means to determine an indication of gaze [18]. This system does not require the ability to see the pupils but is more accurate when more facial features are within scope of the camera [17]. The orientation of the object is determined by solving the Perspective-n-Point (PNP) problem is determining the pose of a set of 3D points and their corresponding 2D projections in the image captured by the camera as shown in Fig. 2. In Fig. 2 the point of interest, P, has a known location in 3D space of (X, Y, Z). When the image is taken by the camera (focal center Fc), the projection of the point of interest, $\mathrm{p}$, is in the $2 \mathrm{D}$ plane by points (u,v). With a known, or estimated, focal center, libraries like OpenCV contain functions to solve these problems to determine the location of the projection point, p, and the Euler angles with the vector highlighted in red [19].

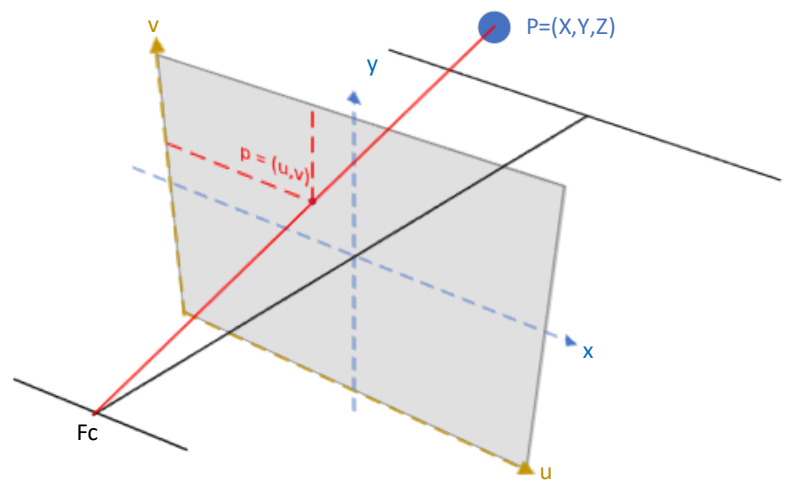

Fig. 2 Diagram of a 3D point in space and the relation with the camera focal point

While the figure shows a single point, this can also be extended to multiple points. However, for the scope of the project, only a single point was needed, and the tip of the nose was selected to determine head pose estimation. The Z-axis of the head pose estimation was extended from the normalized value to be used as a pointer in the overlay interface for controlling the motor or the simulated vehicle. The overlay consisted of four trapezoids that were placed on the outside edges of the image plane as seen in Fig. 3.

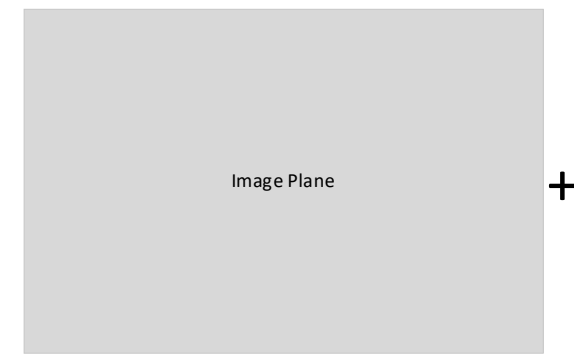

Camera Image

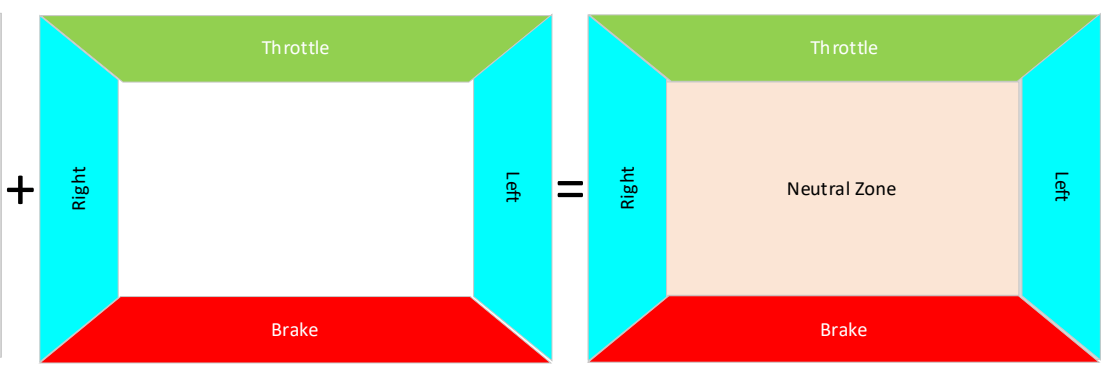

Overlay Sections
Combined Image with Overlay

Fig. 3 The trapezoid pose tracking overlay interface 


\title{
International Journal of Advanced Research in Computer and Communication Engineering
}

\author{
Vol. 9, Issue 8, August 2020
}

\section{DOI 10.17148/IJARCCE.2020.9802}

The coordinates of the projection point are used to determine if the individual is looking at one of the four inputs to the system. When the blink command is received and the projection point is located in one of the four overlay regions, the command of throttle, brake, left, or right is given to the simulation, or for the case of the hardware DC motor, left and right are only accepted. The neutral zone removes the previous command when it is selected when a blink event occurs.

With both the brainwave analysis and pose tracking software in mind, the authors were able to determine the basic layout of the feature. The feature takes advantage of a closed-loop feedback control loop. The subject inputs brainwave and pose tracking data via the NeuroSky EEG sensor and the camera. This data is processed by a controller that will then spin a motor (or steer a simulation). This will cause the vehicle to change the direction that it is currently moving in. The subject will then monitor the vehicle's movement, altering their gaze and blinking to raise the Total Brain Power as needed to steer the vehicle in their determined path. Fig. 4 shows the control loop that the feature will follow.

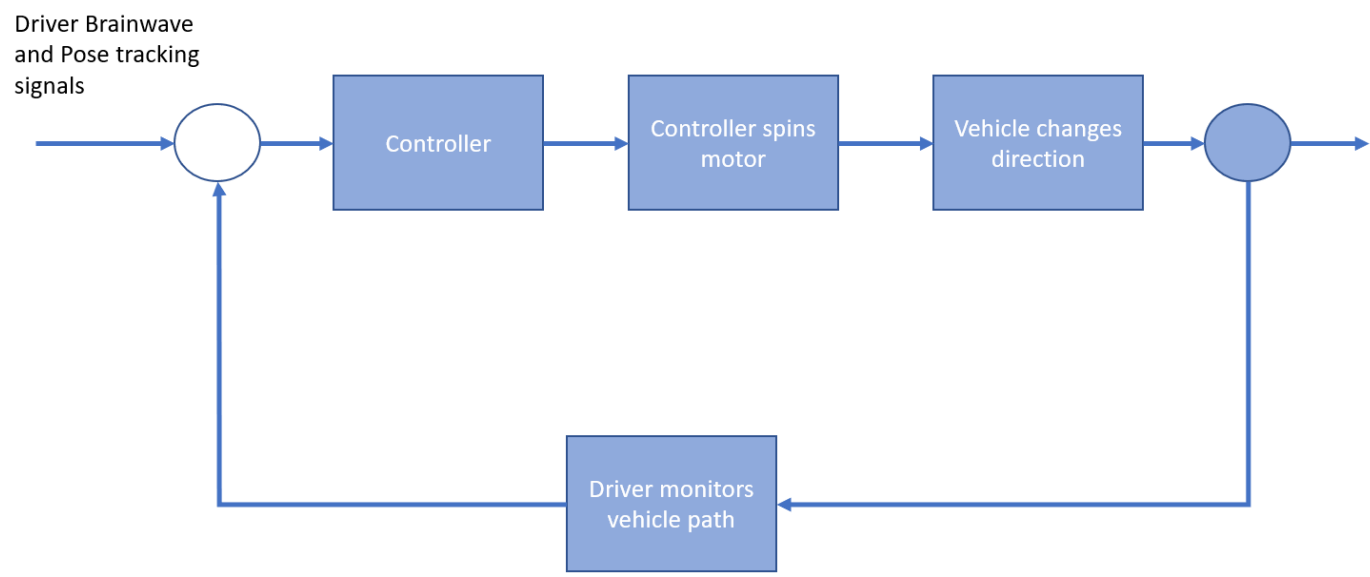

Fig. 4 The feature control loop

Utilizing a closed-loop control system, the feature can continuously adjust the vehicle's path of motion as deemed necessary by the subject. A flowchart view of the feature can be found in Fig. 5. The flowchart utilizes the same basic principles of the closed feedback loop but allows for a step by step understanding of the logic processing that will occur. Once both the EEG headset and the pose tracking software are initialized and data is received, the data will be processed. Processing the data will compare the Total Brain Power to a threshold and determine the gaze direction of the subject. This data will then be passed to both the hardware and the simulation solutions to steer the physical motor and to steer the vehicle in the simulation.

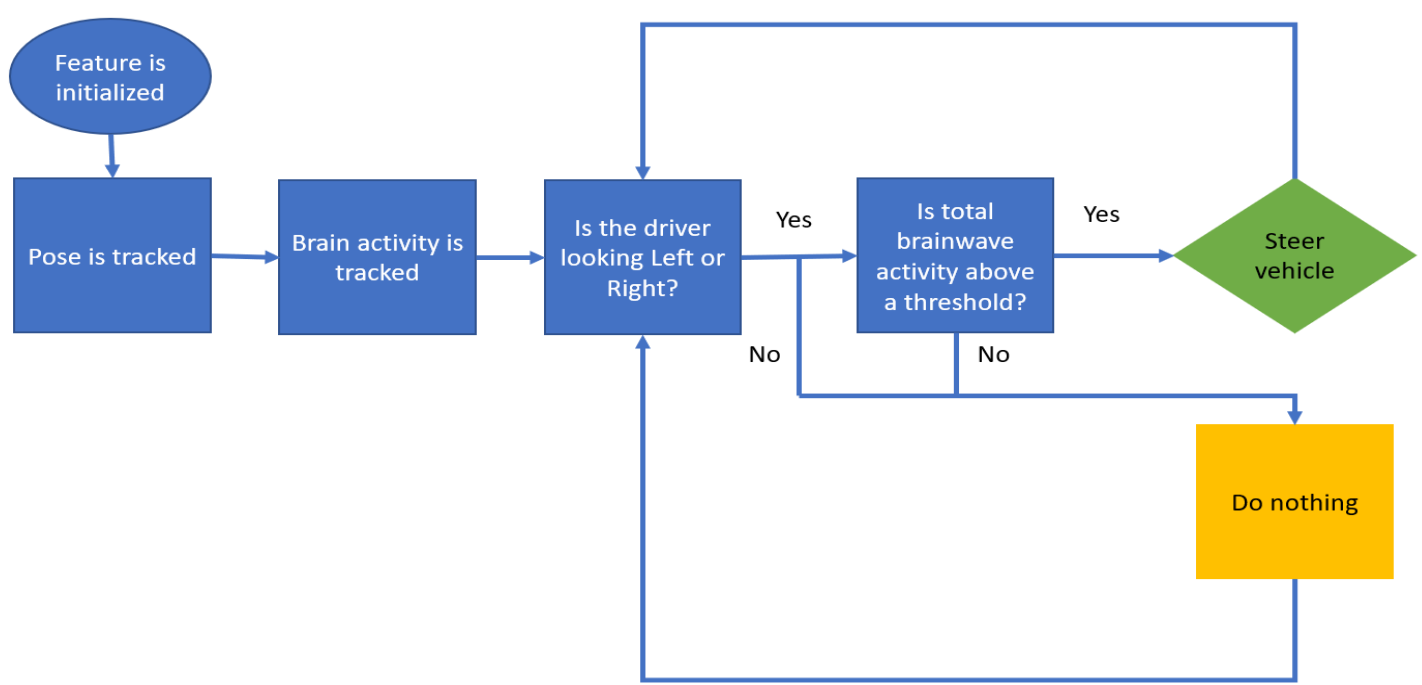

Fig. 5 Flowchart of the pose tracking and brain monitoring software

After determining the feature structure, the components to handle the processing had to be considered. A microcontroller was needed to control the hardware-based solution. A Raspberry Pi with a Linux operating system was chosen due to familiarity with the system and the extensive support available for the Pi. For the simulation solution, a program called 
Car Learning to Act (CARLA) was chosen as one of the authors also had familiarity with the vehicle simulation software. The simulation was chosen to be hosted on a Linux system to create the same messaging structure that would be used to communicate with the Pi. The NeuroSky headset had support for Windows computers. There were attempts to create scripts to intercept the data payload of the headset into a Linux computer, however, the attempts were ultimately unsuccessful. This created a need for a messaging solution to communicate the headset data over to both the hardware and simulation solutions. The solution chose was to utilize the Robotic Operation System (ROS) to build a network of connected devices.

ROS is an open source operating system that is designed to provide a structured communication layer that is not dependent on the hardware of software used on the various hosts in the network [20]. As there are many ways to communicate between computers, ROS provides a means to ensure compatibility between each of the host systems referred to in ROS as nodes. Nodes communicate by passing messages of a strictly defined data type [20].

One of the nodes publishes a message while other nodes that are interested in receiving some or all the data in that message subscribe to receive the message. Each receiving node is then capable of processing the data received to perform a specific action that the node is designed to perform. In this project, one node was the motor spinning node performed by the Raspberry Pi. The other node was the Linux server running the CARLA simulation client. Both nodes subscribed to the same message that was published by a laptop that calculated the head pose estimation, determined if a blink was made, and publishes the data out to the receiving nodes.

The hardware solution for controlling the motor was derived from a previously established project [21]. The Raspberry Pi general purpose input/output (GPIO) pins were used to provide current to a L293D motor driving chip [21]. The chip was used to provide 5 volts in the positive or negative direction to the motor to spin it either clockwise or counterclockwise. A Python script used to drive the motor originated from the project as well, however, it was altered to meet the project needs [21]. The script initializes the used GPIO pins on the Raspberry Pi, begins listening for the ROS message from the Linux virtual machine, and then powers the motor to spin clockwise, counterclockwise, or not at all based on the data received from the ROS message.

CALRA is an open source simulation that was designed to assist in the development and validation of autonomous urban driving [22]. The entire system is built for flexibility and allows engineers and developers to test our designs in an environment that can be controlled and provide for repeatability for various situations. For this project, it provided a simple means to communicate the data from the remote nodes, to the simulation environment to control a vehicle. The commands used to control the vehicle were steering, accelerating, and braking. CALRA developers have also included a bridge to allow communication between the simulation client and a ROS network. This bridge was used to pass the messages from the remote nodes to the simulation client. There were two messages, or topics, that were used to control the vehicle in the simulation environment. These topics provide the ability to enable manual override to control the vehicle, and to control the steering, throttle, and braking of the vehicle. The simulation environment was also modified to allow the user to enable or disable using the mind control input. The simulation environment with pose tracking software can be found in Fig. 6.

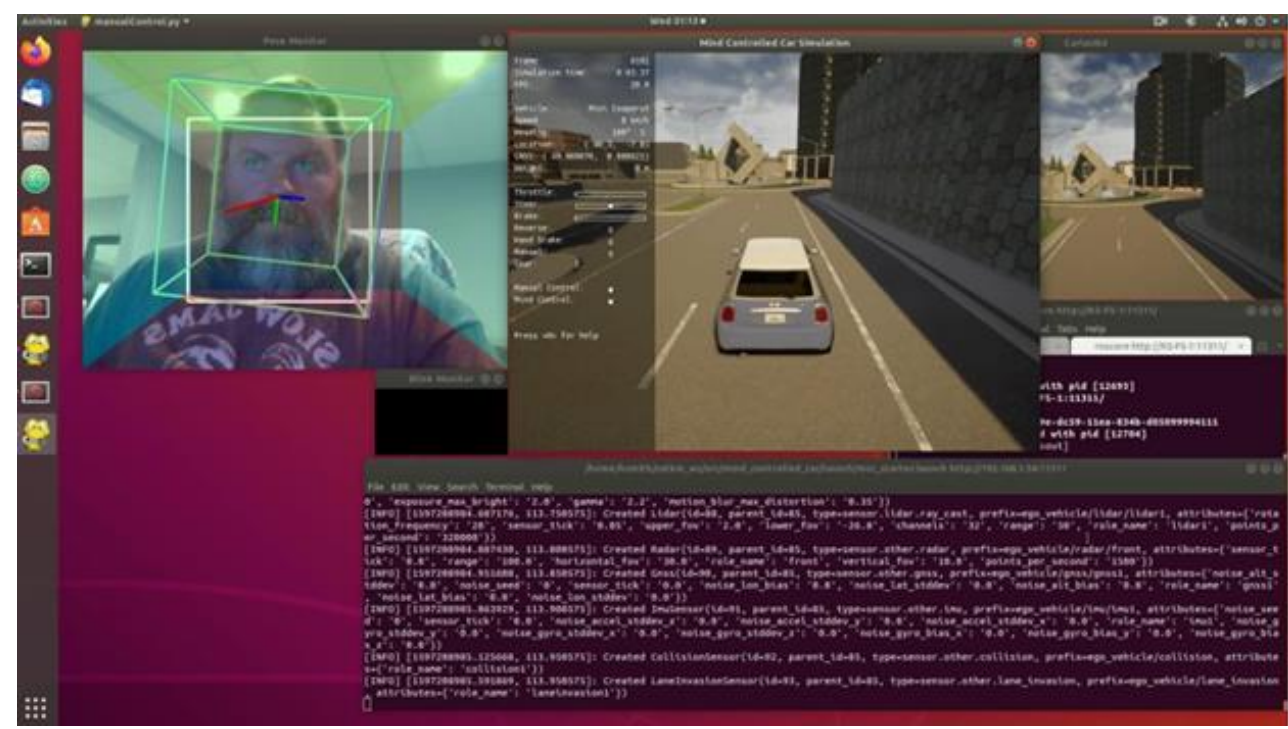

Fig. 6 Pose tracking monitor and CARLA simulated environment 


\section{RESULTS}

An experiment was set up to test if the EEG analysis coupled with pose tracking software could steer a vehicle in the intended direction when desired. For the hardware setup, the subject wore the NeuroSky Mindwave Mobile headset and sat in front of the camera to allow for their pose to be captured. The hardware set up was able to spin a motor in the correct direction when the subject was looking in a non-straightforward direction and their brain activity surpassed the Total Brain Power Threshold. The subject was instructed to blink once approximately every 5 seconds while changing directions that they were looking at in a pattern of left, straight, and then right. The responsiveness of the system was then recorded over a 3-minute timespan. A sample of 30 measurements were recorded to analyze if the system was accurate in determining the direction $\&$ the brain activity of the driver. The sample of the hardware set up can be found in Table 1

Table 1 Sample of test cases

\begin{tabular}{|c|c|c|c|c|c|}
\hline Observation & $\begin{array}{c}\text { Directional } \\
\text { Gaze }\end{array}$ & $\begin{array}{l}\text { Direction } \\
\text { Reported } \\
\end{array}$ & $\begin{array}{c}\text { Total Brain Power } \\
\text { Threshold Exceeded? }\end{array}$ & $\begin{array}{c}\text { Steering } \\
\text { Direction } \\
\end{array}$ & $\begin{array}{c}\text { Correct } \\
\text { Response? }\end{array}$ \\
\hline 1 & Left & Left & Yes & Left & Yes \\
\hline 2 & Straight & Straight & Yes & None & Yes \\
\hline 3 & Right & Right & No & None & No \\
\hline 4 & Left & Left & Yes & Left & Yes \\
\hline 5 & Straight & Straight & No & None & Yes \\
\hline 6 & Right & Right & Yes & Right & Yes \\
\hline 7 & Left & Left & Yes & Left & Yes \\
\hline 8 & Straight & Straight & Yes & None & Yes \\
\hline 9 & Right & Right & Yes & Right & Yes \\
\hline 10 & Left & Left & $\mathrm{No}$ & None & $\mathrm{No}$ \\
\hline 11 & Straight & Straight & Yes & None & Yes \\
\hline 12 & Right & Right & Yes & Right & Yes \\
\hline 13 & Left & Left & No & None & No \\
\hline 14 & Straight & Straight & Yes & None & Yes \\
\hline 15 & Right & Right & Yes & Right & Yes \\
\hline 16 & Left & Left & Yes & Left & Yes \\
\hline 17 & Straight & Straight & Yes & None & Yes \\
\hline 18 & Right & Right & No & None & No \\
\hline 19 & Left & Left & Yes & Left & Yes \\
\hline 20 & Straight & Straight & Yes & None & Yes \\
\hline 21 & Right & Right & No & None & No \\
\hline 22 & Left & Left & Yes & Left & Yes \\
\hline 23 & Straight & Straight & Yes & None & Yes \\
\hline 24 & Right & Right & Yes & Right & Yes \\
\hline 25 & Left & Left & Yes & Left & Yes \\
\hline 26 & Straight & Straight & No & None & Yes \\
\hline 27 & Right & Right & Yes & Right & Yes \\
\hline 28 & Left & Left & No & None & No \\
\hline 29 & Straight & Straight & Yes & None & Yes \\
\hline 30 & Right & Right & Yes & Right & Yes \\
\hline
\end{tabular}

The data of the results shows that the hardware-based system was somewhat accurate. Out of 30 unique measurements, it was able to correctly identify both the direction and the Total Brain Power threshold exceeded 22 times. This success rate of roughly $73 \%$ would not be able to work in a real vehicle setting. The solution steered in the correct direction 24 out of 30 times for an $80 \%$ success rate, however, it benefitted from low brain power levels while the driver was looking straight forward. The directional interpretation was never incorrect during the experiment. No false positives were detected during the testing where the system would determine that the Total Brain Power threshold was exceeded when it was not intended to be exceeded. No false positives are a good sign, however, there may be potential for additional tests to be ran at lower Total Brain Power Thresholds to see if the selected value of 3,000,000 for this project was too high. The simulation acted as a second proof of concept. Initial tests with the simulation the EEG headset proved that the inputs worked as expected and were able to control the vehicle in the simulated environment. However, since the data readings from the EEG headset were updated every second, the vehicle was increasingly difficult to control as the vehicle gained 
speed. To resolve this, the pose tracking software also included a physical keyboard input. Both the keyboard input and the EEG signal provided the same value in the data sent over the ROS topic. This provided the user with the ability to allow for quick corrections in event the EEG values were not received quickly enough. If the data from EEG could be processed faster than the proprietary interface would allow, this additional input would not be required. The throttle commands were also limited to $50 \%$ power to give the user more time to react as the vehicle was accelerating from a stopped position, but maximum speed was still able to be reached. Sending a command while in the neutral zone would remove the throttle and remove any steering input that was presently applied from a previous command. As a result of these modifications, the driver was able to steer the simulated vehicle by looking in the direction of the overlay command sections. Both the hardware solution and the simulation offer the ability to representatively steer a vehicle.

\section{FUTURE RESEARCH}

Future research on similar topics can take away a few lessons learned from this project. The first lesson learned would be to try multiple EEG sensors, specifically ones of a higher quality than the NeuroSky Mindwave Mobile. While the NeuroSky Mindwave Mobile was able to successfully transfer data to a Windows PC that the authors could use, the slow data transfer of roughly one reading per second would make the EEG nearly unusable in a vehicle setting. For reference, some vehicle messages are broadcast over Controlled Area Networks (CAN) at a rate of 10-20 milliseconds [23]. The broadcast rate for similar signals are received anywhere from 50-100 times as frequent as the payload from the EEG headset would be. This delay in data transmission could potentially cause issues in a real time test environment if tested with other vehicle modules present. The data received from the headset was also inconsistent, as previously stated. The authors did not consider multiple headsets for reference in data, so it is not conclusive that the NeuroSky Mindwave Mobile has inconsistent readings, but multiple sensors could be compared to see if one produces more desired results than another. Another consideration for future projects would be to consider different motor types and motor control algorithms. A brushless DC motor was used for testing as the component was affordable and readily available. There was no specific motor algorithm developed for this research. To spin clockwise, voltage was applied in the positive direction. To spin counterclockwise, voltage was applied in the negative direction. Developing a motor algorithm or using different types of motors such as a brushed DC motor or an AC motor, may allow for a more controlled hardware prototype. A more controlled and accurate prototype would be beneficial as this project was able to prove the conceptual question of if this idea is possible. While the results reflect that there is potential for further brainwave analysis coupled with pose tracking software to steer a vehicle, this project did not refine any algorithms used to do so. There is room to improve the responsiveness of the system and to control the motor in more efficient manners such as traveling to specific motor angles or applying specific amounts of torque based on signal input. Integration into a vehicle setting may be considered if future research deems a prototype safe to use in a vehicle. While a simulation is used to prototype the feature, real vehicle use would be another milestone. Calibrating the software to provide a responsive steering output from an electric power steering unit from the EEG headset and pose tracking software would allow for full functional testing of the feature. These future suggestions would be dependent on researchers having both the timeline and the funds to reach the full potential of the feature.

\section{CONCLUSION}

As of the time of writing, the interest in developing brainwave monitoring technology into modern vehicle is a research consideration for multiple automakers [9] [10]. This paper presents the idea of combining EEG and camera tracking to help steer a vehicle. Integrating EEG technology into vehicles may be challenging due to the equipment involved. For both the project presented in this paper and Nissan's "Brain to Vehicle" technology, an EEG headset is used [9]. Incorporating a headset or helmet for features to work in vehicles would require additional driver cooperation than what is given today. Other pursued technologies such as Jaguar's "Sixth Sense" may be easier to integrate as their technology relies on sensors found in the steering wheel to measure brainwave activity [10]. If EEG sensors can be successfully integrated into a vehicle and report accurate data without a headset, the proposed project technology and other technology may have immediate applications. The idea presented in this paper would using the existing idea of EEG based motor control in a different way by using camera tracking as well to determine driver facial position as an additional layer of redundancy. This idea could be further developed to allow for users who may not have the ability to steer a vehicle, such as the many Americans who suffer from conditions causing tetraplegia, who may otherwise would not be able to.

\section{ACKNOWLEDGEMENTS}

Each author equally contributed to this project. Adam Depalma and Matthew Leser each used a headset to study and learn how the data for them is captured and processed. This was used to understand how the brainwave data would be used with the code to control the simulation/motor. Adam and Kevin Smith were responsible for the actual build of the setup 


\title{
International Journal of Advanced Research in Computer and Communication Engineering
}

\author{
Vol. 9, Issue 8, August 2020
}

\section{DOI 10.17148/IJARCCE.2020.9802}

and codes used to drive them. Adam focused on the hardware portion and making the motor spin. Kevin got the simulated environment up and running, as well as developed the code for piloting the vehicle with the headset. Yue Gou was considered the neural expert on this and could help the rest of the team better understand how the EEG worked, as well as what brainwaves to monitor and how. Dr. Dean Aslam took the role of the overall mentor of the project, helping to consult the authors and provide feedback as we progressed through the project. The team all collaborated on this final paper, each taking different sections building it in one paper. The authors are thankful to Brian Wright for providing the NeuroSky headsets from Michigan State University’s ECE101 Lab.

\section{REFERENCES}

[1] "Facts and Figures at a Glance," 2019. [Online]. Available: https://www.nscisc.uab.edu/Public/Facts and Figures 2019 - Final.pdf.

[2] P. Klebine, "What is Tetraplegia? - Spinal Cord Injury Model System," University of Alabama at Birmingham, 2020. [Online]. Available: https://www.uab.edu/medicine/sci/faqs-about-spinal-cord-injury-sci/what-is-tetraplegia. [Accessed 25 August 2020].

[3] "Spinal Cord Injury," 2020. [Online]. Available: https://www.christopherreeve.org/living-with-paralysis/health/causes-of-paralysis/spinal-cordinjury.

[4] “Living With a Spinal Cord Injury," 2019. [Online]. Available: https://www.uofmhealth.org/health-library/ug2580.

[5] D. Vanderwerp, "What Is Power Steering and How Does It Work?," Car and Driver, 2019. [Online]. Available: https://www.caranddriver.com/features/a27888229/power-steering. [Accessed 25 August 2020].

[6] S. K. Pandya, "Understanding brain, mind and soul: Contributions from neurology and neurosurgery," Mens sana monographs, vol. 9, no. 1, p. 129, 2011.

[7] Dean M. Aslam, “Intriguing Aspects of New Scientific Mind Model as EEG Data Based Algorithm,” International Journal of Advanced Research in Computer and Communication Engineering, vol. 8, no. 12, 2019.

[8] "Self-parking car hits the shops," 2003. [Online]. Available: http://news.bbc.co.uk/2/hi/technology/3198619.stm.

[9] J. Stewart, "Nissan Thinks It Can Help You Drive by Reading Your Mind," 2018. [Online]. Available: https://www.wired.com/story/nissan-brainwave-project/.

[10] M. Hussain, “Jaguar's Future Cars Will Sense Your Brainwaves Via 'Sixth Sense' | The Tech Portal,” 2015. [Online]. Available: https://thetechportal.com/2015/06/19/jaguars-future-cars-will-sense-your-brainwaves-via-sixth-sense/.

[11] S. Nacy, S. Kbah, H. Jafer and I. Al-Shaalan, "Controlling a Servo Motor Using EEG Signals from the Primary Motor Cortex," American Journal of Biomedical Engineering, vol. 2016, pp. 139-146, 2016.

[12] V. Rao, "Quadriplegic Driver Drives Car With Sip \& Puff, Head Movement, Voice Controls," 2017. [Online]. Available: https://assistivetechnologyblog.com/2017/08/quadriplegic-drives-car-sip-puff-voice.html.

[13] "EEG Band Power values: Units, Amplitudes, and Meaning," [Online]. Available: http://support.neurosky.com/kb/development-2/eeg-bandpower-values-units-amplitudes-and-meaning.

[14] C. Hennessey, B. Noureddin and P. Lawrence, "A single camera eye-gaze tracking system with free head motion," in Proceedings of the 2006 symposium on Eye tracking research \& applications, 2006.

[15] C. H. Morimoto, A. Amir and M. Flickner, "Free head motion eye gaze tracking without calibration," in CHI'02 extended abstracts on Human factors in computing systems, 2002.

[16] S.-W. Shih, Y.-T. Wu and J. Liu, “A calibration-free gaze tracking technique," in Proceedings 15th International Conference on Pattern Recognition. ICPR-2000, 2000.

[17] E. Murphy-Chutorian and M. M. Trivedi, "Head pose estimation in computer vision: A survey," IEEE transactions on pattern analysis and machine intelligence, vol. 31, no. 4, pp. 607-626, 2008.

[18] E. Murphy-Chutorian, A. Doshi and M. M. Trivedi, "Head pose estimation for driver assistance systems: A robust algorithm and experimental evaluation," in 2007 IEEE Intelligent Transportation Systems Conference, 2007. [19] "Camera
https://docs.opencv.org/2.4/modules/calib3d/doc/camera_calibration_and_3d_reconstruction.html.

[20] M. Quigley, K. Conley, B. Gerkey, J. Faust, T. Foote, J. Leibs, R. Wheeler and A. Y. Ng, "ROS: an open-source Robot Operating System," in ICRA workshop on open source software, 2009.

[21] R. Teja, "Controlling a DC Motor with Raspberry Pi and Python,” 2017. [Online]. Available: https://www.electronicshub.org/controlling-a-dcmotor-with-raspberry-pi/.

[22] A.Dosovitskiy, G.Ros, F.Codevilla, A.Lopez \& V.Koltun, “CARLA: An open urban driving simulator,” arXiv preprint arXiv:1711.03938, 2017

[23] R. Currie, "Hacking the can bus: basic manipulation of a modern automobile through can bus reverse engineering," SANS Institute, 2017. 